\title{
RESEARCH
}

Open Access

\section{CSF synaptic protein concentrations are raised in those with atypical Alzheimer's disease but not frontotemporal dementia}

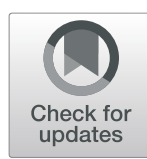

Mica T. M. Clarke', Ann Brinkmalm², Martha S. Foiani ${ }^{3,4}$, lone O. C. Woollacott ${ }^{1}$, Carolin Heller ${ }^{3,4}$, Amanda Heslegrave ${ }^{3,4}$, Ashvini Keshavan ${ }^{1}$, Nick C. Fox ${ }^{1}$, Jonathan M. Schott ${ }^{1}$, Jason D. Warren ${ }^{1}$, Kaj Blennow ${ }^{2,5}$, Henrik Zetterberg ${ }^{2,3,4,5}$ and Jonathan D. Rohrer ${ }^{1 *}$ (D)

\begin{abstract}
Background: Increased CSF levels of a number of synaptic markers have been reported in Alzheimer's disease (AD), but little is known about their concentrations in frontotemporal dementia (FTD). We investigated this in three synaptic proteins, neurogranin, SNAP-25, and synaptotagmin-1.

Methods: CSF samples were analysed from 66 patients with a disorder in the FTD spectrum and 19 healthy controls. Patients were stratified by their tau to $A \beta_{42}$ ratio: those with a ratio of $>1$ considered as having likely $A D$ pathology, i.e. an atypical form of AD ('AD biomarker' group $[n=18]$ ), and $<1$ as likely FTD pathology ('FTD biomarker' group [ $n=48]$ ). A subgroup analysis compared those in the FTD group with likely tau $(n=7)$ and TDP-43 $(n=18)$ pathology. Concentrations of neurogranin were measured using two different ELISAs (Ng22 and Ng36), and concentrations of two SNAP-25 fragments (SNAP-25tot and SNAP-25aa40) and synaptotagmin-1 were measured via mass spectrometry.
\end{abstract}

Results: The AD biomarker group had significantly higher concentrations of all synaptic proteins compared to controls except for synaptotagmin-1 where there was only a trend to increased levels-Ng22, AD mean 232.2 (standard deviation 138.9) pg/ml, controls 137.6 (95.9); Ng36, 225.5 (148.8) pg/ml, 130.0 (80.9); SNAP-25tot, 71.4 (27.9) pM, 53.5 (11.7); SNAP-25aa40, 14.0 (6.3), 7.9 (2.3) pM; and synaptotagmin-1, 287.7 (156.0) pM, 238.3 (71.4). All synaptic measures were significantly higher in the atypical AD group than the FTD biomarker group except for Ng36 where there was only a trend to increased levels—Ng22, 114.0 (117.5); Ng36, 171.1 (75.2); SNAP-25tot, 49.2 (16.7); SNAP-25aa40, 8.2 (3.4); and synaptotagmin-1, 197.1 (78.9). No markers were higher in the FTD biomarker group than controls. No significant differences were seen in the subgroup analysis, but there was a trend to increased levels in those with likely tau pathology.

Conclusions: No CSF synaptic proteins have been shown to be abnormal in those with likely FTD pathologically. Higher CSF synaptic protein concentrations of neurogranin, SNAP-25, and synaptotagmin-1 appear to be related to AD pathology.

Keywords: Alzheimer's disease, Frontotemporal dementia, Synaptic, Biomarkers, Neurogranin, SNAP-25, Synaptotagmin-1

\footnotetext{
* Correspondence: j.rohrer@ucl.ac.uk

'Dementia Research Centre, Department of Neurodegenerative Disease, University College London, Queen Square Institute of Neurology, London WC1N 3BG, UK

Full list of author information is available at the end of the article
}

(c) The Author(s). 2019 Open Access This article is distributed under the terms of the Creative Commons Attribution 4.0 International License (http://creativecommons.org/licenses/by/4.0/), which permits unrestricted use, distribution, and reproduction in any medium, provided you give appropriate credit to the original author(s) and the source, provide a link to the Creative Commons license, and indicate if changes were made. The Creative Commons Public Domain Dedication waiver (http://creativecommons.org/publicdomain/zero/1.0/) applies to the data made available in this article, unless otherwise stated. 


\section{Background}

Frontotemporal dementia (FTD) is a clinically, pathologically, and genetically heterogeneous disorder with few biomarkers that can currently either detect neurodegeneration in the early stages of disease or track disease progression [1]. Synapse dysfunction occurs early in the pathogenesis of neurodegenerative diseases, leading to degradation of vital connections within neuronal networks. Biomarkers of synaptic integrity may therefore represent a potentially more sensitive way of measuring disease onset and severity [2-4].

Most work so far in synaptic biomarkers has focused on cerebrospinal fluid (CSF) where increased protein concentrations are thought to reflect degeneration of functional synapses [2]. In Alzheimer's disease (AD), the concentration of the postsynaptic protein neurogranin, which is involved in synaptic plasticity and enhances the strength of connections, is elevated [2, 5-9], and in amyloid-positive people with mild cognitive impairment, raised neurogranin levels are associated with cognitive decline and conversion to dementia [10]. Only a few studies have investigated other dementias, with concentrations in FTD reported as lower than $\mathrm{AD}[6,8,11,12]$.

Less research has been performed in the measurement of other synaptic proteins in CSF. Synaptosomalassociated protein 25 (SNAP-25) is critical for synaptic vesicle fusion to the membrane, and its concentration has been found to be significantly increased in CSF of those with AD compared to controls [13]. Similarly, CSF concentration of the presynaptic vesicle protein synaptotagmin-1, essential for exocytosis of synaptic vesicles and neurotransmitter release, has also been shown to be significantly increased in people with AD compared to controls [14]. Neither SNAP-25 nor synaptotagmin-1 has been previously investigated in people with FTD.

In this study, we used immunochemical and mass spectrometric methods to measure neurogranin, SNAP25 , and synaptotagmin-1 in CSF to determine whether these synaptic proteins were specific to those with $A D$ pathologically, or whether they were also abnormal in those with likely FTD-related pathology.

\section{Methods}

\section{Participants}

Sixty-six participants with a diagnosis in the FTD and primary progressive aphasia spectrum were consecutively recruited to the University College London FTD cohort studies. These included 21 patients with behavioural variant FTD (bvFTD) [15] and 45 with primary progressive aphasia (PPA) [16]: 11 with semantic variant PPA (svPPA), 16 with non-fluent variant PPA (nfvPPA, 2 of whom had concomitant progressive supranuclear palsy, PSP), 15 with logopenic variant PPA (lvPPA), and 3 with PPA not meeting criteria for a specific variant (here called PPA-not otherwise specified, PPA-NOS). All participants underwent genetic screening, which revealed pathogenic mutations in 10 patients: $4 M A P T, 3$ GRN, and 3 C9orf72 mutations. Nineteen healthy control participants were also recruited. The study was approved by the local ethics committee, and all participants consented to take part.

\section{Measurement of CSF proteins}

All CSF was collected, processed, and stored at $-80^{\circ} \mathrm{C}$ following standardised procedures. Measurement of CSF proteins occurred after all participants had been recruited. The protein concentrations of established biomarkers of CSF total-tau and $\mathrm{A} \beta_{42}$ were measured using sandwich enzyme-linked immunosorbent assays (ELISAs; INNOTEST ${ }^{\oplus}$, Fujirebio Europe N.V., Gent, Belgium) following the manufacturer's instructions.

\section{Immunochemical assays}

Two separate ELISAs were performed for the measurement of neurogranin $(\mathrm{Ng})$ in CSF using in-house generated antibodies: either Ng22 or Ng36 (two different clones, both raised against amino acids 63-75 of $\mathrm{Ng}$ ) as detector, and Ng2 (epitope 52-64) as capture antibody $[12,17]$. We denote the two $\mathrm{Ng}$ measures as $\mathrm{Ng} 22$ and $\mathrm{Ng} 36$ on the basis of the detector antibody clone used.

\section{Mass spectrometry}

High-resolution parallel reaction monitoring was performed on a Q Exactive quadropole-orbitrap mass spectrometer coupled to an Ultimate 3000 chromatography system (Thermo Fisher Scientific) for the parallel quantification of SNAP-25 (amino acids 32-40 = SNAP-25aa40 and Ac-2-16 = SNAP-25tot) and synaptotagmin-1 (amino acids 215-228) in CSF. Antibodies included mouse monoclonal antibody SP12 recognising SNAP-25 and mouse monoclonal antibody SM181 recognising an epitope containing the N-terminally acetylated first 11 amino acids of SNAP-25 (as previously described by Brinkmalm et al. [13]), and monoclonal antibody clone 41.1 recognising the cytoplasmic portion of synaptotagmin-1 from Synaptic Systems (detailed methods previously described by Öhrfelt et al. [14] and Fernström et al. [18]).

\section{Participant stratification}

In the primary analysis, participants with dementia were stratified by their total-tau and $A \beta_{42}$ concentrations into an 'AD biomarker group' (i.e. those likely to have $\mathrm{AD}$ pathologically (atypical AD); tau to $A \beta_{42}$ ratio of $>1$ [19]: $n=18$, mean age at CSF sample collection 66.4 years (standard deviation 5.9), male to female ratio 11:7; 13 patients with lvPPA, 3 with nfvPPA, 1 with svPPA, and 1 with PPA-NOS) and an 'FTD biomarker group' (i.e. those likely to have frontotemporal lobar 
degeneration pathologically; tau to $A \beta_{42}$ ratio of $<1$ : $n=48$, age at CSF 64.0 (6.8), 34:14), and compared with healthy controls (all of whom had a tau to $A \beta_{42}$ ratio of $<1$ ) (Table 1 ).

In a secondary analysis, a subgroup of participants in the FTD biomarker group were stratified according to whether they were likely to have probable TDP-43 pathology $(n=18$; clinical diagnosis of svPPA or FTD with motor neurone disease or genetic diagnosis of a GRN or C9orf72 mutation: age at CSF 62.6 years $(5.6), 13: 5)$ or probable tau pathology $(n=7$; clinical diagnosis of nfvPPA with PSP, genetic diagnosis of a MAPT mutation, and a pathological diagnosis of corticobasal degeneration: age at CSF 64.7 (8.9), 5:2), and compared with healthy controls. Twenty-three of the forty-eight participants in the FTD biomarker group could not be stratified into either group.

\section{Statistical analyses}

All statistical analyses were performed using STATA (v.14). Linear regression analysis with $95 \%$ bias-corrected bootstrapped confidence intervals (CIs) with 1000 repetitions was used to compare concentrations of all synaptic proteins between groups. There was no difference in age between groups, but gender was significantly different between groups and was adjusted for in analyses. Spearman's correlation coefficient was used to investigate the association between each synaptic protein concentration, and between synaptic protein concentrations and both CSF total-tau and $A \beta_{42}$ concentrations.

\section{Data availability}

Data from the study will be available on request.

\section{Results}

\section{Biomarker group stratification}

Concentrations of all synaptic markers were significantly increased in the $\mathrm{AD}$ biomarker group compared to the control group except for synaptotagmin-1 where there was only a trend to higher levels: mean (standard deviation) Ng22, 232.2 (138.9) vs 137.6 (95.9) pg/ml; Ng36, $225.5(148.8)$ vs $130.0(80.9) \mathrm{pg} / \mathrm{ml}$; SNAP-25tot, 71.4 (27.9) vs 53.5 (11.7) pM; SNAP-25aa40, 14.0 (6.3) vs 7.9
(2.3) pM; and synaptotagmin-1, 287.7 (156.0) vs 238.3 (71.4) pM (Fig. 1, Tables 2 and 3).

Furthermore, concentrations of all of the synaptic markers were significantly increased in the AD biomarker group compared to the FTD biomarker group except for Ng36 where there was only a trend to higher levels. Concentrations in the FTD group were as follows: Ng22, 114.0 (117.5) pg/ml; Ng36, $171.1(75.2) \mathrm{pg} / \mathrm{ml}$; SNAP-25tot, 49.2 (16.7) pM; SNAP-25aa40, 8.2 (3.4) pM; and synaptotagmin-1, 197.1 (78.9) pM.

No significant differences were seen between the FTD biomarker group and the controls.

\section{Probable pathology stratification}

There were no significant differences in the comparison of the likely tau and TDP-43 groups with controls: Ng22, 169.0 (151.3) vs 103.1 (95.3) pg/ml; Ng36, 179.8 (47.2) vs 151.1 (75.9) pg/ml; SNAP-25tot, 56.2 (13.0) vs 50.6 (13.6) pM; SNAP-25aa40, 9.2 (2.0) vs 7.9 (2.1) pM; and synaptotagmin-1, 214.4 (65.3) vs 220.7 (85.0) pM (Fig. 2, Tables 4 and 5).

No significant differences in CSF synaptic protein concentrations were seen in the direct comparison between those with likely tau and TDP-43 pathology. However, there was a trend to an increased concentration of markers in the tau group compared to the TDP-43 group in all but synaptotagmin-1.

\section{Correlation with other fluid biomarkers}

There were significant correlations between all synaptic marker concentrations except between $\mathrm{Ng} 36$ and synaptotagmin-1 (Fig. 3). In general, correlations were lower between Ng36 and the other markers.

All synaptic biomarkers, except Ng36, were significantly positively correlated with CSF total-tau concentrations in the FTD biomarker group (Ng22 $r=0.57$; SNAP-25tot $r=$ 0.71 ; SNAP-25aa40 $r=0.54$; synaptotagmin- $r=0.68$; all $p<0.0001$ ) and AD biomarker group (Ng22 $r=0.86$; SNAP25tot $r=0.77$; SNAP-25aa40 $r=0.73$; synaptotagmin- $r=$ 0.88 ; all $p<0.01$ ). The concentrations of both SNAP-25 measures and synaptotagmin-1 were significantly positively correlated with CSF $\mathrm{A} \beta_{42}$ concentrations in the FTD biomarker group (SNAP-25tot $r=0.32$; SNAP-25aa40 $r=0.35$;

Table 1 Baseline characteristics of the cohort

\begin{tabular}{|c|c|c|c|c|c|c|c|c|}
\hline $\begin{array}{l}\text { Participant } \\
\text { stratification }\end{array}$ & Number & $\begin{array}{l}\text { Age at CSF } \\
(\text { mean (SD)) }\end{array}$ & Gender (M:F) & $\begin{array}{l}\text { CSF total-tau } \\
(\mathrm{pg} / \mathrm{ml} \text {, mean } \\
(\mathrm{SD}))\end{array}$ & $\begin{array}{l}\text { CSF } A \beta_{42} \\
(\mathrm{pg} / \mathrm{ml}, \\
\text { mean (SD)) }\end{array}$ & $\begin{array}{l}\text { Total-tau to } \\
\mathrm{A} \beta_{42} \text { ratio }\end{array}$ & $\begin{array}{l}\text { Disease duration } \\
(\text { mean }(\mathrm{SD}))\end{array}$ & $\begin{array}{l}\text { MMSE } \\
(\text { mean (SD)) }\end{array}$ \\
\hline Control & 19 & $64.2(6.9)$ & $10: 9$ & $327.9(93.2)$ & $1012.7(237.3)$ & $0.3(0.1)$ & N/A & N/A \\
\hline AD biomarker group & 18 & $66.4(5.9)$ & $11: 7$ & $984.4(543.6)$ & $471.1(179.0)$ & $2.5(2.0)$ & $3.5(2.0)$ & $21.1(5.2)$ \\
\hline FTD biomarker group & 48 & $64.0(6.8)$ & $34: 14$ & $376.1(173.3)$ & $851.2(246.1)$ & $0.5(0.2)$ & $5.7(4.1)$ & $23.9(7.0)$ \\
\hline Probable TDP-43 pathology & 18 & $62.6(5.6)$ & $13: 5$ & $456.0(226.0)$ & $863.4(222.5)$ & $0.5(0.3)$ & $6.6(4.9)$ & $23.3(6.7)$ \\
\hline Probable tau pathology & 7 & $64.7(8.9)$ & $5: 2$ & $444.4(146.4)$ & $894.3(214.1)$ & $0.5(0.2)$ & $5.5(3.1)$ & $21.1(10.4)$ \\
\hline
\end{tabular}



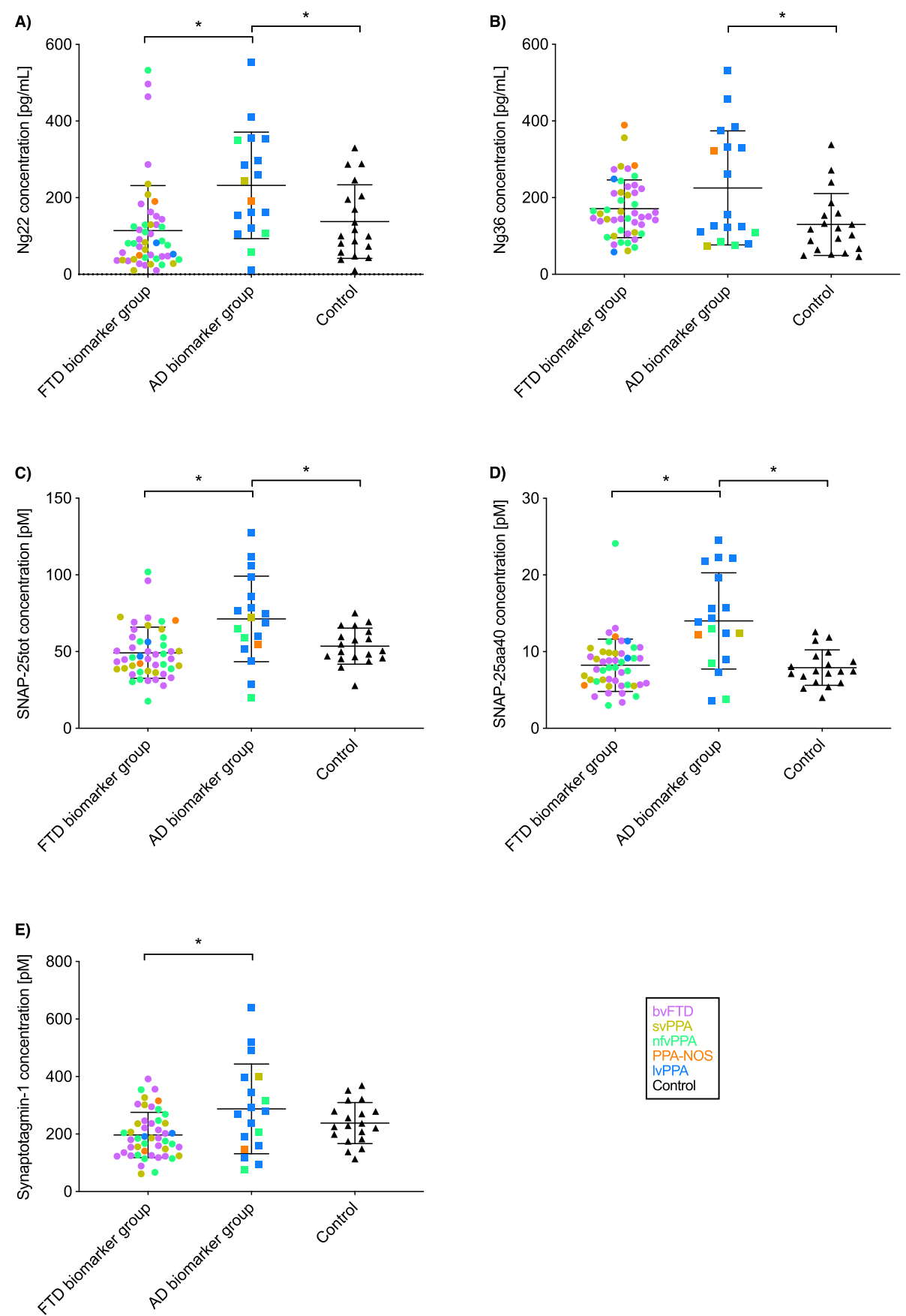

Fig. 1 Concentrations for the five synaptic measures in the AD biomarker group, FTD biomarker group, and controls: a Ng22, b Ng36, c SNAP25tot, $\mathbf{d}$ SNAP-25aa40, and e synaptotagmin-1. Within each group, data points are coloured to represent the clinical phenotype of each participant: purple $=$ bvFTD, yellow $=$ svPPA, green $=$ nfvPPA, blue $=$ IVPPA, orange $=$ PPA-NOS, and black $=$ controls

synaptotagmin-1 $r=0.33$; all $p<0.05)$ but not in the AD biomarker group.

\section{Discussion}

We show that the synaptic proteins measured in this study are elevated in those with likely AD pathologically (i.e. atypical $\mathrm{AD}$ ) but not in those with probable FTD pathology. Elevated levels of synaptic proteins in CSF are thought to reflect loss of synapses and therefore decreased concentration in the brain as proteins leak into the surrounding fluid. This pattern is well reported in $\mathrm{AD}$, particularly for neurogranin but also for SNAP-25 and synaptotagmin-1 [2, 4, 5, 7-9, 1214]. This study confirms those findings in a group 
Table 2 CSF protein concentrations of each synaptic measure for each biomarker group

\begin{tabular}{llll}
\hline Synaptic measure & FTD biomarker group (mean (SD)) & AD biomarker group (mean (SD)) & Control group (mean (SD)) \\
\hline Ng22 $(\mathrm{pg} / \mathrm{ml})$ & $114.0(117.5)$ & $232.2(138.9)$ & $137.6(95.9)$ \\
Ng36 $(\mathrm{pg} / \mathrm{ml})$ & $171.1(75.2)$ & $225.5(148.8)$ & $130.0(80.9)$ \\
SNAP-25tot $(\mathrm{pM})$ & $49.2(16.7)$ & $71.4(27.9)$ & $53.5(11.7)$ \\
SNAP-25aa40 $(\mathrm{pM})$ & $8.2(3.4)$ & $14.0(6.3)$ & $7.9(2.3)$ \\
Synaptotagmin-1 $(\mathrm{pM})$ & $197.1(78.9)$ & $287.7(156.0)$ & $238.3(71.4)$ \\
\hline
\end{tabular}

with an atypical form of AD (mainly the logopenic variant of PPA).

It is unclear whether increased synaptic protein concentrations in CSF in AD but not FTD reflect the topography of volume loss, with anatomical targets of $\mathrm{AD}$ including areas rich in particular proteins, or instead the pathological process underlying AD $[6,9]$. Neurogranin is mainly expressed in the cortex, amygdala, and hippocampus and enhances synaptic plasticity, critical for long term potentiation in the hippocampus and therefore learning and memory [2, 12, 20, 21]. Increased expression in regions affected in AD (i.e. hippocampus and parietal and temporal cortices) may explain the elevated levels of neurogranin in CSF from people with AD but not FTD, with greater synapse loss in these areas. However, in one previous study comparing neurogranin concentrations in typical and atypical AD, concentrations were higher in atypical AD (posterior cortical atrophy) than in controls, but higher still in amnestic AD [9]. Along with a lack of correlation between neurogranin concentrations and regional volumes, this suggests that the specificity of increased neurogranin to AD may be related to both the underlying pathogenesis of $\mathrm{AD}$ itself, as well as the anatomical involvement. Less is known about the anatomical distribution of SNAP-25 and synaptotagmin-1, although there appears to be relatively diffuse expression throughout the cortex [22-24], again suggestive that there may be some specific association with AD pathology rather than topography.

Synaptic markers were significantly correlated with total-tau concentration in both biomarker groups. Totaltau is significantly increased in the CSF of individuals with $\mathrm{AD}$ and to a lesser extent in those with FTD [25].
This correlation with total-tau has been reported elsewhere for each of the synaptic biomarkers, though not in FTD $[2,8,13,14]$, and is likely to represent the association of both total-tau and the synaptic biomarker concentration with the extent of neurodegeneration. Additionally, in the FTD biomarker group only, presynaptic SNAP-25 and synaptotagmin-1 concentrations correlated with $\mathrm{A} \beta_{42}$ concentration. In previous studies, the same correlation has been found with SNAP-25 and synaptotagmin- 1 in controls but not in $\mathrm{AD}$ (where $\mathrm{A} \beta_{42}$ concentration is reduced) $[13,14]$. It is unclear what this correlation represents, although a similar association with $A \beta_{42}$ in an FTD group has been shown with other proteins [26].

In the present study, we also stratified participants by their probable frontotemporal lobar degeneration pathology to investigate any relationship with the underlying proteinopathy. While there were no significant differences in synaptic concentrations between those with probable tau and TDP-43 pathology, there was a trend to higher concentrations in the tau group across most synaptic biomarkers. Interestingly, one recent study revealed a positive correlation between CSF neurogranin concentration and postmortem tau neurofibrillary tangle pathology [12], suggesting a specific association with AD-tau pathology. Further work is therefore needed in larger groups with underlying non-AD tauopathies, as there may be specific associations of synaptic protein concentrations with particular disorders.

The lack of elevation of CSF synaptic protein concentrations in FTD biomarker group remains unexplained, particularly as synaptic dysfunction is well described in FTD [27]. As discussed above, this may represent the

Table 3 Adjusted mean differences and 95\% confidence intervals in CSF protein concentrations of the five synaptic measures between biomarker groups.

\begin{tabular}{llllll}
\hline Group comparison & $\mathrm{Ng} 22(\mathrm{pg} / \mathrm{ml})$ & $\mathrm{Ng} 36(\mathrm{pg} / \mathrm{ml})$ & SNAP-25tot $(\mathrm{pM})$ & SNAP-25aa40 (pM) & Synaptotagmin-1 (pM) \\
\hline FTD vs AD biomarker group & $-117.7(-193.8,-54.2)$ & $-54.0(-124.0,10.3)$ & $-21.9(-35.6,-8.0)$ & $-5.7(-8.7,-2.6)$ & $-88.6(-168.0,-18.9)$ \\
FTD biomarker vs control group & $-22.7(-78.4,33.6)$ & $41.7(-1.7,84.0)$ & $-3.9(-11.4,4.2)$ & $0.5(-1.1,2.3)$ & $-37.4(-80.4,2.9)$ \\
AD biomarker vs control group & $95.0(10.5,169.6)$ & $95.7(20.7,173.3)$ & $18.0(2.5,32.4)$ & $6.2(2.9,9.4)$ & $51.2(-30.3,130.5)$ \\
\hline
\end{tabular}



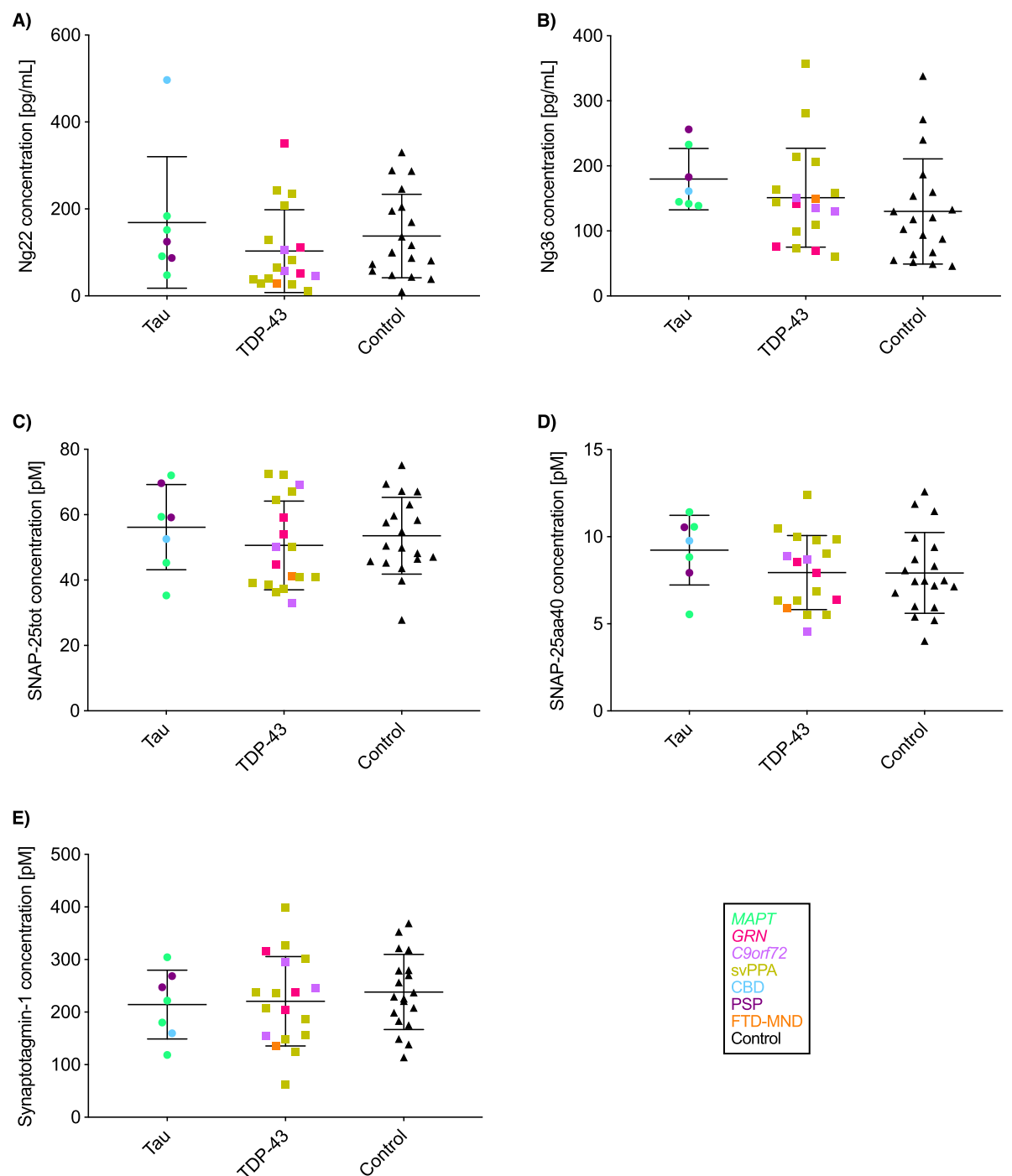

Fig. 2 Concentrations for the five synaptic measures in patients with probable tau and TDP-43 pathology and controls: a Ng22, b Ng36, c SNAP25tot, d SNAP-25aa40, and e synaptotagmin-1. Within each group, data points are coloured to represent the reason for allocation to each probable pathology group: green $=$ MAPT mutation carrier, blue $=$ pathologically confirmed $C B D$, dark purple $=$ concomitant PSP, orange $=$ concomitant MND, yellow = sVPPA, pink = GRN mutation carrier, light purple = C9orf72 mutation carrier, and black = controls

anatomical distribution of particular synaptic proteins measured (suggesting investigation of other markers that are expressed more widely across the cortex) or the specificity of their loss to the AD pathological process. It could also represent the extent of dysfunction (e.g. being of lower magnitude in FTD compared with AD, suggesting improvement in the sensitivity of current assays may be important). Another issue is that synaptic proteins are processed into fragments before being released into CSF [10, 21], and therefore, future assays should investigate alternative fragments of these proteins.

Table 4 CSF protein concentrations of each synaptic measure for those with likely tau and TDP-43 pathology

\begin{tabular}{llll}
\hline Synaptic measure & Tau (mean (SD)) & TDP-43 (mean (SD)) & Control (mean (SD)) \\
\hline Ng22 (pg/ml) & $169.0(151.3)$ & $103.1(95.3)$ & $137.6(95.9)$ \\
Ng36 $(\mathrm{pg} / \mathrm{ml})$ & $179.8(47.2)$ & $151.1(75.9)$ & $130.0(80.9)$ \\
SNAP-25tot (pM) & $56.2(13.0)$ & $50.6(13.6)$ & $53.5(11.7)$ \\
SNAP-25aa40 (pM) & $9.2(2.0)$ & $7.9(2.1)$ & $7.9(2.3)$ \\
Synaptotagmin-1 (pM) & $214.4(65.3)$ & $220.7(85.0)$ & $238.3(71.4)$ \\
\hline
\end{tabular}


Table 5 Adjusted mean differences and 95\% confidence intervals in CSF protein concentrations of the five synaptic measures between the likely tau and TDP-43 groups, and controls

\begin{tabular}{llllll}
\hline Group comparison & $\mathrm{Ng} 22(\mathrm{pg} / \mathrm{ml})$ & $\mathrm{Ng} 36(\mathrm{pg} / \mathrm{ml})$ & SNAP-25tot $(\mathrm{pM})$ & SNAP-25aa40 (pM) & Synaptotagmin-1 (pM) \\
\hline Tau vs TDP-43 group & $65.9(-14.9,246.4)$ & $28.6(-22.7,69.8)$ & $5.6(-6.2,15.2)$ & $1.3(-0.4,2.7)$ & $-6.4(-67.8,47.8)$ \\
Tau vs control group & $32.7(-45.3,216.6)$ & $51.5(-0.7,101.7)$ & $2.8(-8.8,12.5)$ & $1.4(-0.5,3.0)$ & $-21.9(-79.5,33.7)$ \\
TDP-43 vs control group & $-33.2(-97.6,37.8)$ & $23.0(-26.0,78.5)$ & $-2.8(-10.9,6.7)$ & $0.2(-1.5,1.6)$ & $-15.4(-67.7,37.5)$ \\
\hline
\end{tabular}

No significant differences were seen

The limitations of this study include the relatively small sample size, especially of the FTD subgroups with probable tau and TDP-43 pathology. Additionally, the atypical AD group only included those with PPA clinically, particularly those with lvPPA, although patients with a bvFTD phenotype have also been reported to have underlying $\mathrm{AD}$ pathology on occasion [28]. Replication of these findings in a larger cohort would strengthen interpretation of our findings. Moreover, although we have reported a specificity of synaptic biomarkers to $\mathrm{AD}$ consistent with the existing literature, this study is the first to explore SNAP-25 and synaptotagmin-1 in FTD and verification is needed from independent cohorts. The cross-sectional design limits our evaluation of these synaptic proteins as prognostic biomarkers. Longitudinal measurements would enable investigations of CSF synaptic concentrations over time and whether these relate to cognitive performance and structural loss, which are important endpoints for clinical trials.

\section{Conclusions}

In conclusion, none of the CSF synaptic proteins investigated here have been shown to be abnormal in those with likely FTD pathologically. Higher CSF synaptic protein concentrations of neurogranin, SNAP-25, and

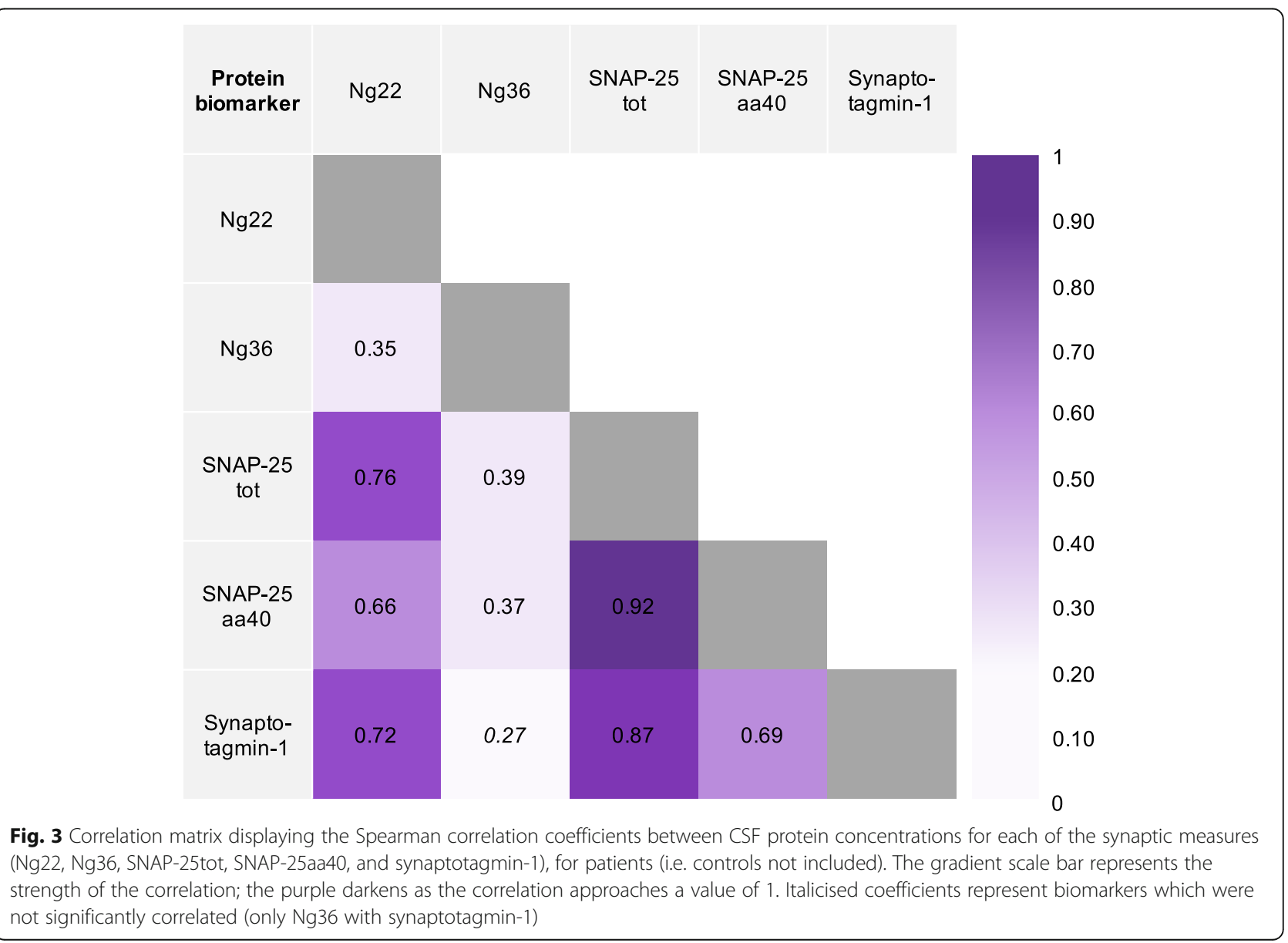


synaptotagmin-1 appear to be related to $\mathrm{AD}$ pathology. Biomarkers that track changes in pathophysiological events such as synapse dysfunction will be useful in the identification of novel therapeutic targets, or indeed markers to measure the effects of emerging therapies. The synaptic proteins presented here provide candidate biomarkers for disease pathogenesis in $\mathrm{AD}$, though the search for synaptic biomarkers of FTD continues.

\section{Abbreviations}

AD: Alzheimer's disease; bvFTD: Behavioural variant frontotemporal dementia; CBD: Corticobasal degeneration; Cl: Confidence interval; CSF: Cerebrospinal fluid; FTD: Frontotemporal dementia; IvPPA: Logopenic variant primary progressive aphasia; nfvPPA: Non-fluent variant primary progressive aphasia; Ng: Neurogranin; PPA: Primary progressive aphasia; PPA-NOS: Primary progressive aphasia-not otherwise specified; PSP: Progressive supranuclear palsy; SD: Standard deviation; SNAP-25: Synaptosomal-associated protein 25; SVPPA: Semantic variant primary progressive aphasia

\section{Acknowledgements}

Not applicable.

\section{Statistical analysis}

Conducted by Mica Clarke, MSc, University College London.

\section{Authors' contributions}

MTMC analysed and interpreted the data and was a major contributor in writing the manuscript. $A B$ performed the mass spectrometric analyses. All authors read and approved the final manuscript.

\section{Funding}

The Dementia Research Centre is supported by Alzheimer's Research UK, Brain Research Trust, and The Wolfson Foundation. This work was supported by the NIHR Queen Square Dementia Biomedical Research Unit, the NIHR UCL/H Biomedical Research Centre and the Leonard Wolfson Experimental Neurology Centre (LWENC) Clinical Research Facility as well as an Alzheimer's Society grant (AS-PG-16-007). MTMC is supported by a Brain Research UK PhD studentship. JDR is supported by an MRC Clinician Scientist Fellowship (MR/M008525/1) and has received funding from the NIHR Rare Disease Translational Research Collaboration (BRC149/NS/MH).

\section{Availability of data and materials}

The datasets analysed during the current study will be available from the corresponding author on reasonable request.

\section{Ethics approval and consent to participate}

The study was approved by the local ethics committee, and all participants consented to take part.

\section{Consent for publication}

This manuscript does not contain individual or identifiable data.

\section{Competing interests}

The authors declare that they have no competing interests.

\section{Author details}

'Dementia Research Centre, Department of Neurodegenerative Disease, University College London, Queen Square Institute of Neurology, London WC1N 3BG, UK. ${ }^{2}$ Department of Psychiatry and Neurochemistry, Institute of Neuroscience and Physiology, The Sahlgrenska Academy at the University of Gothenburg, Mölndal, Sweden. ${ }^{3}$ UK Dementia Research Institute at UCL, London, UK. ${ }^{4}$ Department of Neurodegenerative Disease, University College London, London, UK. ${ }^{5}$ Clinical Neurochemistry Laboratory, Sahlgrenska University Hospital, Mölndal, Sweden.
Received: 13 August 2019 Accepted: 4 December 2019

Published online: 17 December 2019

\section{References}

1. Meeter $L H$, Kaat $L D$, Rohrer JD, van Swieten JC. Imaging and fluid biomarkers in frontotemporal dementia. Nat Rev Neurol. 2017;13(7):406-19. https://doi.org/10.1038/nrneurol.2017.75.

2. Thorsell A, Bjerke M, Gobom J, Brunhage E, Vanmechelen E, Andreasen N, et al. Neurogranin in cerebrospinal fluid as a marker of synaptic degeneration in Alzheimer's disease. Brain Res. 2010;1362:13-22. https:/doi. org/10.1016/j.brainres.2010.09.073.

3. Blennow, K., \& Zetterberg, H. (2015). The past and the future of Alzheimer's disease CSF biomarkers-a journey toward validated biochemical tests covering the whole spectrum of molecular events. Front Neurosci, 9(SEP), 1-8. doi: https://doi.org/10.3389/fnins.2015.00345.

4. Goetzl EJ, Kapogiannis D, Schwartz JB, Lobach IV, Goetzl L, Abner EL, et al. Decreased synaptic proteins in neuronal exosomes of frontotemporal dementia and Alzheimer's disease. FASEB J. 2016;30(12):4141-8. https://doi. org/10.1096/fj.201600816R.

5. De Vos A, Jacobs D, Struyfs H, Fransen E, Andersson K, Portelius E, et al. Cterminal neurogranin is increased in cerebrospinal fluid but unchanged in plasma in Alzheimer's disease. Alzheimer's and Dementia. 2015;11(12):14619. https://doi.org/10.1016/j.jalz.2015.05.012.

6. Lista S, Toschi N, Baldacci F, Zetterberg H, Blennow K, Kilimann I, et al. Cerebrospinal fluid neurogranin as a biomarker of neurodegenerative diseases: a cross-sectional study. J Alzheimers Dis. 2017;59(4):1327-34. https://doi.org/10.3233/JAD-170368.

7. Mattsson N, Insel PS, Palmqvist S, Portelius E, Zetterberg H. Cerebrospinal fluid tau, neurogranin, and neurofilament light in Alzheimer's disease. EMBO Mol Med. 2016;8(10):1184-96. https://doi.org/10.15252/emmm.

8. Wellington H, Paterson RW, Portelius E, Törnqvist U, Magdalinou N, Fox NC, et al. Increased CSF neurogranin concentration is specific to Alzheimer disease. Neurology. 2016;86(9):829-35. https://doi.org/10.1212/WNL. 0000000000002423.

9. Wellington H, Paterson RW, Suárez-González A, Poole T, Frost C, Sjöbom U, et al. CSF neurogranin or tau distinguish typical and atypical Alzheimer disease. Ann Clin Transl Neurol. 2018;5(2):162-71. https://doi.org/10.1002/ acn3.518.

10. Kvartsberg H, Duits FH, Ingelsson M, Andreasen N, Öhrfelt A, Andersson K, et al. Cerebrospinal fluid levels of the synaptic protein neurogranin correlates with cognitive decline in prodromal Alzheimer's disease. Alzheimers Dementia. 2015;11(10):1180-90. https://doi.org/10.1016/j.jalz. 2014.10.009.

11. Janelidze S, Hertze J, Zetterberg H, Landqvist M. Cerebrospinal fluid neurogranin and YKL-40 as biomarkers of Alzheimer's disease; 2015. p. 1220. https://doi.org/10.1002/acn3.266.

12. Portelius $E$, Olsson B, Höglund $\mathrm{K}$, Cullen $\mathrm{NC}$, Kvartsberg $\mathrm{H}$, Andreasson $\mathrm{U}$, et al. Cerebrospinal fluid neurogranin concentration in neurodegeneration: relation to clinical phenotypes and neuropathology. Acta Neuropathol. 2018;0123456789:1-14. https://doi.org/10.1007/s00401-018-1851-x.

13. Brinkmalm A, Brinkmalm G, Honer WG, Frölich L, Hausner L, Minthon L, et al. SNAP-25 is a promising novel cerebrospinal fluid biomarker for synapse degeneration in Alzheimer's disease. Mol Neurodegener. 2014;9: 53. https://doi.org/10.1186/1750-1326-9-53.

14. Öhrfelt A, Brinkmalm A, Dumurgier J, Brinkmalm G, Hansson O, Zetterberg $\mathrm{H}$, et al. The pre-synaptic vesicle protein synaptotagmin is a novel biomarker for Alzheimer's disease. Alzheimers Res Ther. 2016;8(1):1-10. https://doi.org/10.1186/s13195-016-0208-8.

15. Rascovsky K, Hodges JR, Knopman D, Mendez MF, Kramer JH, Neuhaus J, et al. Sensitivity of revised diagnostic criteria for the behavioural variant of frontotemporal dementia0. Brain. 2011;134(9):2456-77. https://doi.org/10. 1093/brain/awr179.

16. Gorno-Tempini ML, Hillis AE, Weintraub S, Kertesz A, Mendez M, Cappa SF, et al. Classification of primary progressive aphasia and its variants. Neurology. 2011;76(11):1006-14. https://doi.org/10.1212/WNL. ob013e31821103e6.

17. Becker B, Nazir FH, Brinkmalm G, Camporesi E, Kvartsberg H, Portelius E, et al. Alzheimer-associated cerebrospinal fluid fragments of neurogranin are generated by Calpain-1 and prolyl endopeptidase. Mol Neurodegener. 2018; 13(1):47. https://doi.org/10.1186/s13024-018-0279-z. 
18. Fernström E, Minta K, Andreasson U, Sandelius $\AA$, Wasling P, Brinkmalm A, et al. Cerebrospinal fluid markers of extracellular matrix remodelling, synaptic plasticity and neuroinflammation before and after cranial radiotherapy. J Intern Med. 2018;284(2). https://doi.org/10.1111/joim.12763.

19. Paterson R, Slattery C, Poole T, Nicholas J, Magdalinou N, Toombs J, et al. Cerebrospinal fluid in the differential diagnosis of Alzheimer's disease: clinical utility of an extended panel of biomarkers in a specialist cognitive clinic. Alzheimers Res Ther. 2018;10(32). https://doi.org/10.1186/s13195-018-0361-3.

20. Represa A, Deloulme JC, Sensenbrenner M, Ben-Ari Y, Baudier J. Neurogranin: immunocytochemical localization of a brain-specific protein kinase C substrate. J Neurosci. 1990;10(12):3782-92. https://doi.org/10.1523/ JNEUROSCI.10-12-03782.1990

21. Zetterberg H, Blennow K. Neurogranin levels in cerebrospinal fluid: a new addition to the Alzheimer disease diagnostic toolbox. JAMA Neurology. 2015;72(11):1237-8. https://doi.org/10.1001/jamaneurol.2015.2075.

22. Bogdanovic N, Davidsson P, Gottfries J, Volkman I, Winblad B, Blennow K. Regional and cellular distribution of synaptic proteins in the normal human brain. Brain Aging. 2002;2(2):18-30.

23. Glavan G, Schliebs R, Zivin M. Synaptotagmins in neurodegeneration. Anat Rec. 2009;292:1849-62. https://doi.org/10.1002/ar.21026.

24. Yamamori S, Itakura M, Sugaya D, Katsumata O, Sakagami H, Takahashi M. Differential expression of SNAP-25 family proteins in the mouse brain. J Comp Neurol. 2010;519(5):916-32. https://doi.org/10.1002/cne.22558.

25. Sjogren M, Sjögren M, Davidsson P. Tullberg M, Minthon L, Wallin A, et al. Both total and phosphorylated tau are increased in Alzheimer's disease. J Neurol Neurosurg Psychiatry. 2001;70(5):624-30. https://doi.org/10.1136/ jnnp.70.5.624.

26. Woollacott I, Nicholas JM, Heslegrave A, Heller C, Foiani M, Dick K, et al. Cerebrospinal fluid solumble TREM2 levels in frontotemporal dementia differ by genetic and pathological subgroup. Alzheimers Res Ther. 2018; 10(79):1-14. https://doi.org/10.1186/s13195-018-0405-8.

27. Starr A, Sattler R. Synaptic dysfunction and altered excitability in C9orf72 ALS/FTD. Brain Res. 2018;1693(A):98-108. https://doi.org/10.1016/j.brainres. 2018.02.011

28. Singleton E, Pijnenburg Y, Sudre C, Groot C, Kochova E, Barkhof F, et al. Investigating the clinico-anatomical dissociation in the behavioural variant of Alzheimer's disease; 2019. https://doi.org/10.1101/19006676.

\section{Publisher's Note}

Springer Nature remains neutral with regard to jurisdictional claims in published maps and institutional affiliations.

Ready to submit your research? Choose BMC and benefit from:

- fast, convenient online submission

- thorough peer review by experienced researchers in your field

- rapid publication on acceptance

- support for research data, including large and complex data types

- gold Open Access which fosters wider collaboration and increased citations

- maximum visibility for your research: over $100 \mathrm{M}$ website views per year

At $\mathrm{BMC}$, research is always in progress.

Learn more biomedcentral.com/submissions 